\title{
An Iterative Interpolation De-convolution Algorithm for Super-resolution Land Cover Mapping
}

\author{
Feng Ling, Giles M. Foody, Fellow, IEEE, Yong Ge, Member, IEEE, Xiaodong Li and Yun Du
}

\begin{abstract}
Super-resolution mapping (SRM) is a method to produce a fine spatial resolution land cover map from coarse spatial resolution remotely sensed imagery. A popular approach for SRM is a two-step algorithm, which first increases the spatial resolution of coarse fraction images by interpolation, and then determines class labels of fine resolution pixels using the maximum a posteriori (MAP) principle. By constructing a new image formation process that establishes the relationship between observed coarse resolution fraction images and the latent fine resolution land cover map, it is found that the MAP principle only matches with area-to-point interpolation algorithms, and should be replaced by de-convolution if an area-to-area interpolation algorithm is to be applied. A novel iterative interpolation deconvolution (IID) SRM algorithm is proposed. The IID algorithm first interpolates coarse resolution fraction images with an areato-area interpolation algorithm, and produces an initial fine resolution land cover map by de-convolution. The fine spatial resolution land cover map is then updated by re-convolution, back-projection and de-convolution iteratively until the final result is produced. The IID algorithm was evaluated with simulated shapes, simulated multi-spectral images, and degraded Landsat images, including comparison against three widely used SRM algorithms: pixel swapping, bilinear interpolation, and Hopfield neural network. Results show that the IID algorithm can reduce the impact of fraction errors, and can preserve the patch continuity and the patch boundary smoothness, simultaneously. Moreover, the IID algorithm produced fine resolution land cover maps with higher accuracies than those produced by other SRM algorithms.
\end{abstract}

Index Terms - Interpolation, De-convolution, Super-resolution Mapping

\section{INTRODUCTION}

$\mathrm{S}$ uper-resolution mapping (SRM) is a method to predict the spatial distribution of land cover classes located within the geographical area represented by coarse spatial resolution pixels. SRM is generally regarded as a post-processing analysis of spectral unmixing, in which the coarse spatial resolution fraction images produced by spectral unmixing are used to produce a fine spatial resolution land cover map [1, 2].

Manuscript received ***. This work was supported in part by the National Basic Research Program of China under Grant 2013cb733205, in part by the State Key Laboratory of Resources and Environmental Informational System of China and in part by the Wuhan ChenGuang Youth Sci.\&Tech. Project under Grant 2014072704011254

F. Ling, X. Li and Y. Du are with Key Laboratory of Monitoring and Estimate for Environment and Disaster of Hubei Province, Institute of Geodesy
Compared with hard classification that only produces a land cover map at the coarse spatial resolution pixel scale and spectral unmixing that only produces coarse spatial resolution fraction images without detailed land cover spatial pattern information, SRM can produce a more informative result. Presently, SRM has been shown to be a promising solution to the mixed pixel problem that is commonly encountered with coarse spatial resolution remotely sensed images, and has been widely applied in many research fields successfully [3-7].

The input to a SRM analysis is typically a set of coarse spatial resolution fraction images, in which the image value represents the area percentage of one land cover class in one coarse spatial resolution pixel. The output of SRM is a fine spatial resolution land cover map, that is, a labeled image in which the label represents the land cover class that a fine spatial resolution pixel belongs to. Given that the input of SRM is continuous values (e.g. percentage class coverage) while the output is discrete values (e.g. hard class labels), and their spatial resolutions are different, SRM often needs perform two tasks: the increment of the spatial resolution of input fraction images and the transformation between continuous and discrete values.

Various SRM algorithms have been proposed in the last decades, and different strategies are applied for these two tasks of SRM [7-21]. Commonly, the two tasks of SRM are completed in two steps [22,23]. The first step is to increase the spatial resolution. In this step, the input coarse spatial resolution fraction images are magnified to fine spatial resolution images. The second step is to determine the class label using the fine spatial resolution images produced in the first step. This twostep approach is often referred as interpolation-based SRM [22], because an interpolation algorithm is often used in the first step to increase the spatial resolution of input fraction images. Critically, an objective of the first step is estimating the class membership probabilities for each fine spatial resolution pixel. The latter are used in the second step to determine the class label of the fine spatial resolution pixels. Although several different methods have been proposed for the second step, the maximum

and Geophysics, Chinese Academy of Sciences, Wuhan 430077, (e-mail: lingf@whigg.ac.cn).

G. M. Foody is with the School of Geography, University of Nottingham, NG7 2RD Nottingham, U.K.

Y. Ge is with Institute of Geographical Sciences \& Natural Resources Research, State Laboratory of Resources \& Environmental Information System, Chinese Academy of Sciences. 
a posteriori (MAP) principle is widely used and so the class label is assigned as the land cover class with the highest probability of occurrence for each fine resolution pixel.

The use of the MAP principle is intuitively attractive but its suitability may be limited by the method used to interpolate coarse resolution fraction images into fine resolution images. A variety of interpolation algorithms are available [22-29], but may vary in suitability for SRM using the MAP principle.

A key feature to note is that the fraction value in the input coarse spatial resolution images is an areal value which represents the proportion of the pixel's area that is covered with a certain land cover class. Often the fraction value is also taken to be the probability of a coarse spatial resolution pixel belonging to a certain land cover class [30]. The interpolation process is used to estimate the class membership probabilities for each point or fine resolution pixel contained within the area of a coarse resolution pixel. If the MAP principle is to be used, this interpolation should produce a probability value for each fine resolution pixel. That allows the MAP principle to be applied to class membership probability data that relate to each single fine resolution pixel alone, independent of the situation for other fine resolution pixels. Consequently, from the range of interpolation methods available [31], an interpolation method, which can transform the input coarse resolution fraction (or probability) values to the fine resolution probability values, should be used.

Several area-to-point interpolation algorithms have been suggested for use in SRM, by considering the fine resolution probability value as a point value. For example, the indicator cokriging algorithm, a geo-statistical method that can account for the differences between the areal data and the point predictions may be suitable for this application [26, 27]. Another approach, which considers interpolation as a regression problem and uses the support vector regression to learn the relationship between areal data and point predictions, has also been proposed [29]. However, for these area-to-point interpolation algorithms, additional information at the fine spatial resolution is required prior to the interpolation. For example, to use the indicator cokriging algorithm, the variogram model must be extracted from existing fine spatial resolution land cover map. Similarly, for the support vector regression algorithm, a fine spatial resolution land cover map is needed to generate the fine/coarse spatial resolution patch pairs.

As the required additional information is often unavailable, area-to-point interpolation algorithms are difficult to use and alternative spatial interpolation algorithms have been used in SRM. In particular, common area-to-area spatial interpolation algorithms have been used in SRM [22]. For example, Verhoeye and De Wulf used the kriging interpolation algorithm [24], Mertens et al. used the spatial attraction algorithm [25], Wang et al. used the edge-directed interpolation algorithm [32], Wang and Shi applied the bilinear and bicubic interpolation algorithms [28], and Chen et al. applied the high-accuracy surface modeling interpolation algorithm [33]. These interpolation algorithms are especially attractive as they estimate values for fine spatial resolution pixels without additional fine spatial resolution information. Generally, for a fine spatial resolution pixel, the interpolated value is estimated by averaging the surrounding coarse resolution fraction values with a weighting approach, and is considered as a fine resolution probability value. Since the interpolated values would often be strongly correlated with the real probability values of fine spatial resolution pixels, it is possible for an acceptable land cover map to emerge. However, the interpolated value itself is indeed a coarse resolution probability value, but not a fine resolution probability value, as no additional process is applied for transforming their different spatial resolutions. Therefore, a consequence of the use of areato-area interpolation and the MAP principle is that the output map may contain speckle-like and linear artifacts caused by mismatches between both probability values with different spatial resolutions. The latter can degrade the value of the map and may place a requirement for additional post-processing to refine the resulting fine spatial resolution land cover map [24, 34].

Theoretically, the performance of the two-step SRM algorithm should be affected not only by the first interpolation step and the second label assigning step, but also the interaction of both steps. Focusing on the label assignment step, existing algorithms mostly adopt the MAP principle to assign class labels for fine spatial resolution pixels, although there are some minor differences in implementation details. When the MAP principle is applied, however, class membership probabilities must be real probability values of individual fine spatial resolution pixel. In this situation, the area-to-point interpolation algorithm should be applied in the interpolation step as an areato-area interpolation algorithm is unsuitable because the interpolated values are not fine resolution probability values but coarse resolution probability values. In other words, if an areato-area spatial interpolation algorithm is used in the first step of two-step SRM algorithms, the MAP principle should not be applied in the second step. Other more suitable principles should be used to replace the MAP principle in recognition of the nature of the interpolated coarse resolution probability value.

Given the simplicity of the area-to-area spatial interpolation algorithms, we aim to improve existing interpolation-based two-step SRM algorithms by modifying the MAP principle used in the label assignment step. This is based on a conceptual image formation process that models how coarse spatial resolution fraction images are produced from a fine spatial resolution land cover map. Based on the image formation process, a novel iterative interpolation de-convolution SRM algorithm is proposed. In the proposed algorithm, the traditional MAP principle is replaced by the de-convolution process, which matches coarse resolution probability values produced by area-to-area interpolation algorithms. An iterative process is further applied in the proposed algorithm in order to reinforce information included in input fraction images into the resulting fine spatial resolution land cover map. Thus, this article aims to substantially enhance SRM by proposing a completely new approach that addresses fundamental concerns and tests it relative to established state-of-the-art methods. The rest of the paper is organized as follows: Section II discusses the 
conceptual image formation process. The proposed algorithm is explained in detail in Section III and its performance is validated through several experiments in Section IV. Section V concludes the paper.

\section{CONCEPTUAL IMAge Formation PROCESS}

Assumed that a remotely sensed image includes $M \times N$ coarse spatial resolution pixels, and that the number of land cover classes in the entire image is $C$. Let $\mathbf{Y}=\left[\mathbf{Y}_{1}, \mathbf{Y}_{2}, \ldots, \mathbf{Y}_{C}\right]$ be the fraction images yielded by spectral unmixing, where $\mathbf{Y}_{c}$ is the fraction image of the $c^{\text {th }}$ class. By setting the zoom factor as $z$ and using $\mathbf{Y}$ as input, SRM aims to generate a labeled fine spatial resolution land cover map $\mathbf{X}$ that includes $(z \times M) \times(z \times N)$ pixels, by dividing each coarse spatial resolution pixel $V$ into $z \times z$ fine spatial resolution pixels. All these fine spatial resolution pixels are considered pure pixels and should be assigned to a specific land cover class as $x_{v} \in(1, \ldots C)$ for the fine spatial resolution pixel $v$.

SRM aims to estimate the latent fine spatial resolution land cover map $\mathbf{X}$ from observed coarse spatial resolution fraction images $\mathbf{Y}$. This process can be considered as an inversion problem, and SRM can then be considered as an inversion model. In order to better understand the inversion process, we aim to establish a forward model as opposed to the inversion model. The forward model is the image formation process that is used to describe how a particular coarse spatial resolution fraction images $\mathbf{Y}$ can be generated if the latent fine spatial resolution land cover map $\mathbf{X}$ is known.

Fig. 1 illustrates the conceptual image formation process. Fig. 1(a) is a fine spatial resolution land cover map including two classes shown as white and black. Because each land cover class has its own coarse spatial resolution fraction image, the original fine spatial resolution land cover map is divided into individual fine spatial resolution images for each land cover class. The value in the individual fine spatial resolution image is an indicator value, where 1 means that the fine spatial resolution pixel belongs to this class, and 0 means that the fine spatial resolution pixel belongs to the other class. Thus, for the black and white classes, their individual fine spatial resolution indicator images are shown in Fig. 1(b) and Fig. 1(c), respectively.

For each land cover class, the process used to produce the coarse resolution fraction image from the fine spatial resolution indicator image consists of two steps: convolution and decimation.

The first step in the conceptual image formation process is convolution, with which the fine spatial resolution indicator image is convoluted to produce a new fine spatial resolution fraction image. The spatial resolution of the convoluted fraction image is the same as that of the fine spatial resolution indicator image, while the convoluted value becomes a mixture of indicator pixel values within a local area defined by the convolution kernel. This process is similar to 'blurring' in the field of image analysis, where the observed image pixel value is a mixture of itself and the surrounding pixels subject to the camera's Point Spread Function (PSF). Given that each coarse spatial resolution pixel corresponds to $z \times z$ fine spatial resolution pixels for SRM, the convoluted value should correspond to the observed fraction value, and be calculated as the percentage of the fine spatial resolution pixels assigned to the class $c$ in the coarse spatial resolution pixel $V$ [35]:

$$
f_{c}(V)=\sum_{v \in V} \mathbf{X}_{c}(v) / z^{2}
$$

where $\mathbf{X}_{c}$ is the fine spatial resolution indicator image of the class $c$, and $\mathbf{X}_{c}(v)$ is the indicator value of the fine spatial resolution pixel $v \cdot f_{c}(V)$ is the convoluted value, which is also the fraction value, of the class $c$ in the coarse spatial resolution pixel $V$.

Thus, if we set the PSF, also the convolution kernel, as a $z \times z$ matrix $\mathbf{H}$, whose element values are all $1 / z^{2}$, the convoluted fine spatial resolution fraction image can be calculated as:

$$
\mathbf{F}_{c}=\mathbf{X}_{c} \otimes \mathbf{H}
$$

where $\otimes$ means the convolution process. In the example, Fig. 1(d) and Fig. 1(e) are the results of convolution for Fig. 1(b) and Fig. 1(c), respectively.

The second step in the conceptual image formation process is decimation. The size of the observed coarse spatial resolution fraction image is $M \times N$, while the size of the convoluted fine spatial resolution fraction image is $(z \times M) \times(z \times N)$. Then, the observed coarse spatial resolution only includes $1 / z^{2}$ values of the convoluted fine spatial resolution fraction image. The decimation process extracts the central pixel value within every $z \times z$ pixels from the convoluted fine spatial resolution fraction image (highlighted in Figs. 1(d) and 1(e)). Each extracted fraction value corresponds to an observed fraction value in a coarse spatial resolution pixel, and all values form the coarse spatial resolution fraction images. In the example, Fig. 1(f) and Fig. 1(g) are the results of decimation for Fig. 1(d) and Fig. 1(e), respectively. The decimation process can be expressed as:

$$
\mathbf{Y}_{c}=\left(\mathbf{F}_{c}\right) \downarrow^{z}
$$

where $\downarrow^{z}$ means the decimation process with the zoom factor $z$.

By considering all land cover classes, the whole conceptual image formation process is expressed as:

$$
\mathbf{Y}=(\mathbf{X} \otimes \mathbf{H}) \downarrow^{z}
$$

\section{MethodOLOGY}

If both aforementioned convolution and decimation steps in the conceptual image formation process are inverted, the latent fine spatial resolution land cover map can be produced from observed coarse spatial resolution fraction images. As shown in Fig. 1, the inverse process of decimation is interpolation, and the inverse process of convolution is de-convolution. Therefore, the SRM process can be performed by first interpolating the observed coarse spatial resolution fraction images and then deconvolving the interpolated fine spatial resolution fraction images. From this viewpoint, an iterative interpolation deconvolution (IID) SRM algorithm is proposed. The framework 


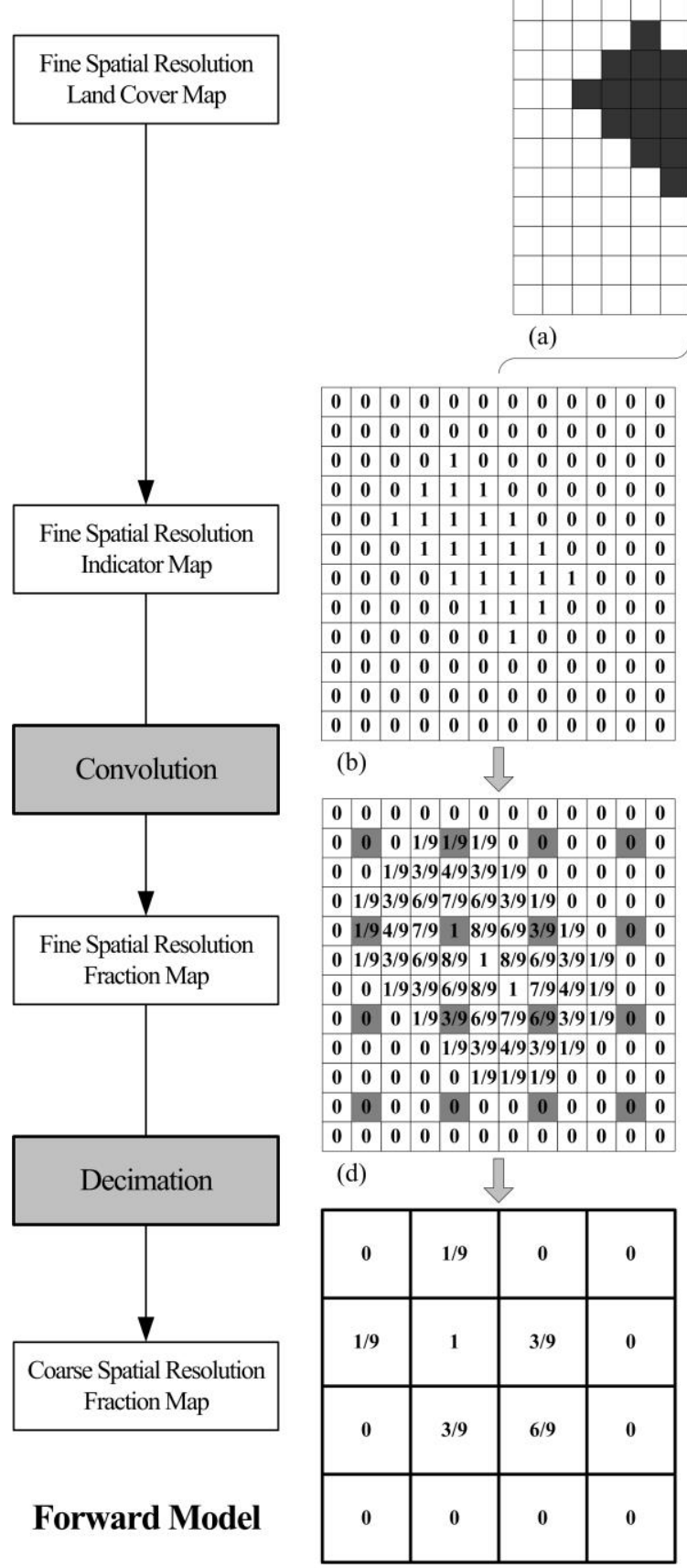

(f)

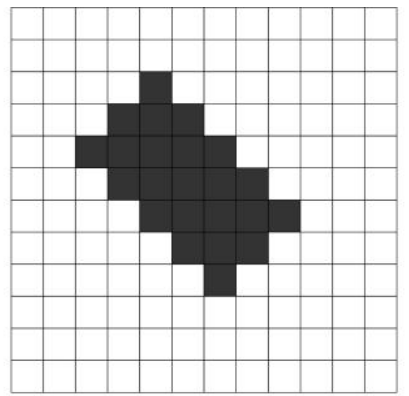

(a)

\begin{tabular}{|l|l|l|l|l|l|l|l|l|l|l|l|}
\hline 1 & 1 & 1 & 1 & 1 & 1 & 1 & 1 & 1 & 1 & 1 & 1 \\
\hline 1 & 1 & 1 & 1 & 1 & 1 & 1 & 1 & 1 & 1 & 1 & 1 \\
\hline 1 & 1 & 1 & 1 & 0 & 1 & 1 & 1 & 1 & 1 & 1 & 1 \\
\hline 1 & 1 & 1 & 0 & 0 & 0 & 1 & 1 & 1 & 1 & 1 & 1 \\
\hline 1 & 1 & 0 & 0 & 0 & 0 & 0 & 1 & 1 & 1 & 1 & 1 \\
\hline 1 & 1 & 1 & 0 & 0 & 0 & 0 & 0 & 1 & 1 & 1 & 1 \\
\hline 1 & 1 & 1 & 1 & 0 & 0 & 0 & 0 & 0 & 1 & 1 & 1 \\
\hline 1 & 1 & 1 & 1 & 1 & 0 & 0 & 0 & 1 & 1 & 1 & 1 \\
\hline 1 & 1 & 1 & 1 & 1 & 1 & 0 & 1 & 1 & 1 & 1 & 1 \\
\hline 1 & 1 & 1 & 1 & 1 & 1 & 1 & 1 & 1 & 1 & 1 & 1 \\
\hline 1 & 1 & 1 & 1 & 1 & 1 & 1 & 1 & 1 & 1 & 1 & 1 \\
\hline 1 & 1 & 1 & 1 & 1 & 1 & 1 & 1 & 1 & 1 & 1 & 1 \\
\hline
\end{tabular}

(c)

$\sqrt{3}$

\begin{tabular}{|l|l|l|l|l|l|l|l|l|l|l|l|}
\hline 1 & $\mathbf{1}$ & $\mathbf{1}$ & $\mathbf{1}$ & $\mathbf{1}$ & $\mathbf{1}$ & $\mathbf{1}$ & $\mathbf{1}$ & $\mathbf{1}$ & $\mathbf{1}$ & $\mathbf{1}$ & $\mathbf{1}$ \\
\hline & 1 & 1 & & & & & & & & &
\end{tabular}

\begin{tabular}{|llllll|l|l|l|ll|}
\hline 1 & 1 & 1 & $8 / 98 / 9$ & $8 / 9$ & 1 & 1 & 1 & 1 & 1 & 1 \\
\hline
\end{tabular}

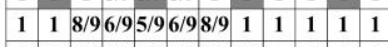

\begin{tabular}{llll|l|l|l|}
\hline & $8 / 96 / 93 / 9$ & $2 / 93 / 96 / 98 / 9$ & 1 & 1 & 1 & 1 \\
\hline
\end{tabular}

\begin{tabular}{|l|l|l|l|l|l|l|l|}
\hline 1 & $8 / 95 / 92 / 9$ & 0 & $1 / 93 / 96 / 98 / 9$ & 1 & 1 & 1 \\
\hline 1 & $8 / 96 / 93 / 9$ & $1 / 9$ & 0 & $1 / 93 / 96 / 98$ & & & \\
\hline
\end{tabular}

\begin{tabular}{llllllll|l} 
& 1 & $8 / 96 / 93 / 9$ & $1 / 9$ & 0 & $1 / 93 / 96 / 98 / 9$ & 1 & 1
\end{tabular}

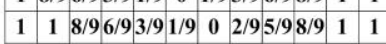

\begin{tabular}{|llllllll}
\hline & 1 & 1 & $8 / 96 / 93 / 92 / 93 / 96 / 98 / 9$ & 1 & 1 \\
\hline
\end{tabular}

\begin{tabular}{|l|l|l|l|l|l|l|l|}
\hline 1 & 1 & 1 & 1 & $8 / 96 / 95 / 96 / 98 / 9$ & 1 & 1 & 1 \\
\hline
\end{tabular}

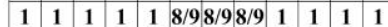

\begin{tabular}{llllllllllllll}
\hline 1 & 1 & 1 & 1 & 1 & 1 & 1 & 1 & 1 & 1 & 1 & 1 \\
\hline
\end{tabular}

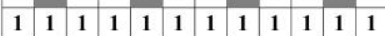

(e)

\begin{tabular}{|c|c|c|c|}
\hline 1 & $8 / 9$ & 1 & 1 \\
\hline $8 / 9$ & 0 & $6 / 9$ & 1 \\
\hline 1 & $6 / 9$ & $3 / 9$ & 1 \\
\hline 1 & 1 & 1 & 1 \\
\hline
\end{tabular}

(g)

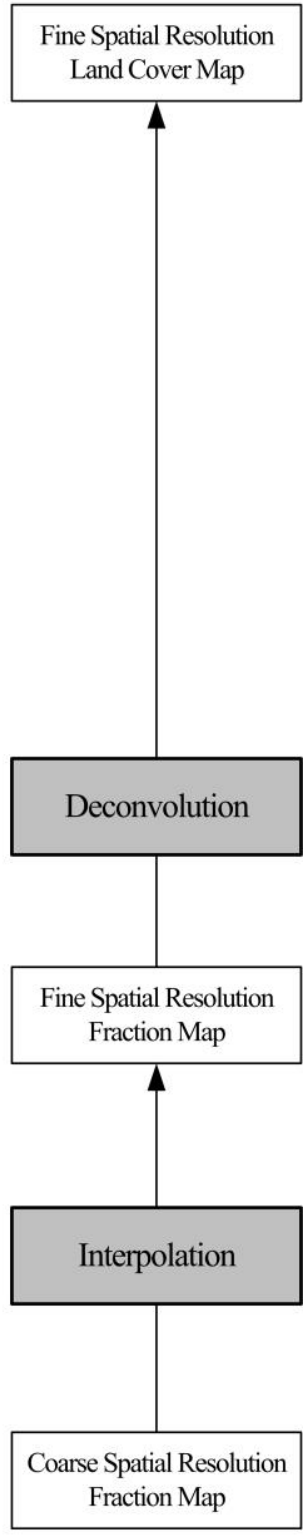

Inversion Model

Fig. 1. An example shows the conceptual image formation process including the forward model and the inversion model. The zoom factor $\mathrm{z}=3$. (a) is the fine spatial resolution land cover map including pixels and two classes as black and white; (b) and (c) are fine spatial resolution indicator images for the black and white classes, respectively. The indicator value 1 means that the fine spatial resolution pixel belongs to this class, and 0 means that the fine spatial resolution pixel belongs to other classes. (d) and (e) are convoluted fine spatial resolution fraction images, where the value of a target fine spatial resolution pixel is calculated by averaging the values of fine spatial resolution pixels, using the target pixel as the central in the corresponding indicator image. (f) and (g) are coarse spatial resolution fraction images produced by decimation, that is, extracting the central fraction value for each coarse spatial resolution pixel including fine spatial resolution pixels from the convoluted fine spatial resolution fraction images.

of the proposed IID algorithm is shown in Figure 2. The input of IID is the observed coarse spatial resolution fraction images $\mathbf{Y}$. For each land cover class, the fraction image is initially interpolated to generate a fine spatial resolution fraction image F, whose spatial resolution is the same as that of the final fine resolution land cover map. These initially interpolated fine spatial resolution fraction images $\mathbf{F}$ are then used to generate an intermediate fine spatial resolution land cover map $X$, which is iteratively updated by convolution and back projection procedures until the final land cover map $\mathrm{X}$ is produced. The key steps of IID are discussed in detail as follows. 


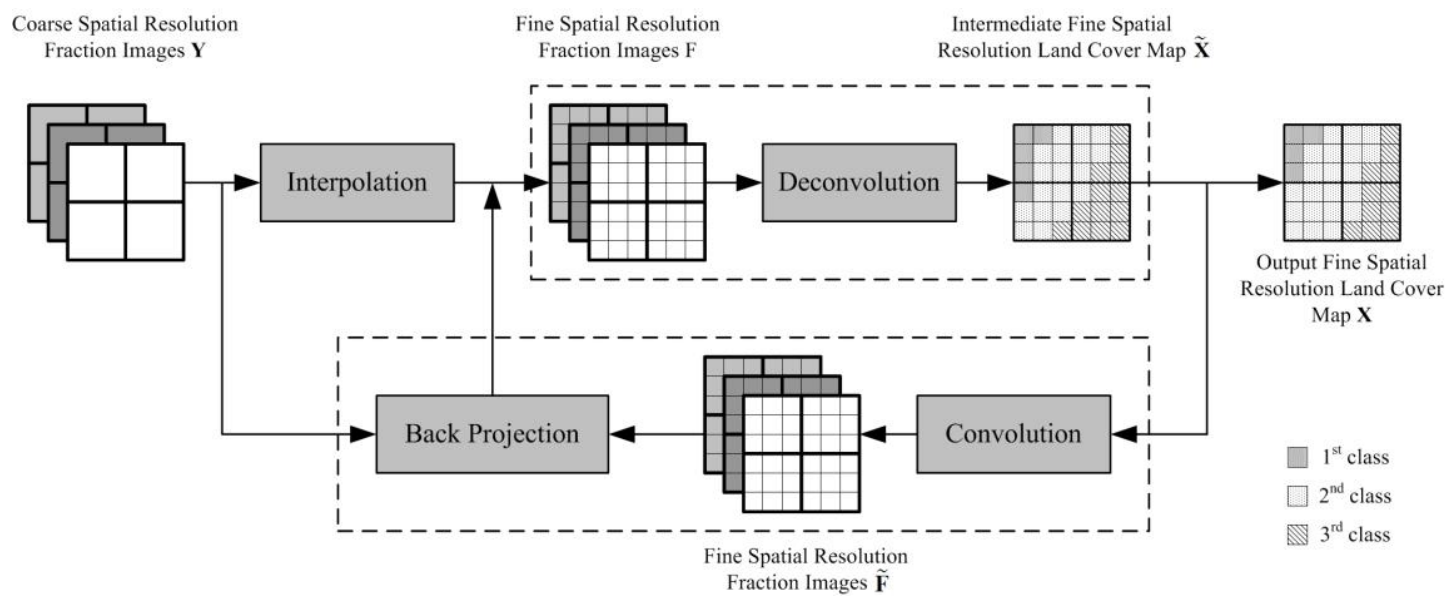

Fig. 2. The flowchart of the proposed iterative interpolation de-convolution SRM algorithm. The fraction image includes $2 \times 2$ coarse spatial resolution pixels. The zoom factor $\mathrm{z}=3$. The fine resolution land cover map ( $\mathbf{X}$ and $\mathbf{X}$ ) includes 3 land cover classes.

\section{A. Interpolation}

Interpolation is the inversion process of decimation in the conceptual image formation process. It aims to estimate the fine resolution fraction image that includes $(z \times M) \times(z \times N)$ values from the observed coarse resolution image that includes $M \times N$ values. All values in the fine resolution fraction image are estimated by interpolating the observed fractional values with an 'area-to-area' spatial interpolation algorithm. Different spatial interpolation algorithms, such as bilinear, cubic or kriging, may produce dissimilar results. The selection of the interpolation algorithm is, however, beyond the scope of this paper.

\section{B. De-convolution}

Once all fine spatial resolution fraction images for different land cover classes have been produced by interpolation, a fine spatial resolution land cover map can then be produced by deconvolution, the inversion process of convolution. The input to the de-convolution analysis is the interpolated fine spatial resolution fraction images, which are convoluted versions of individual indicator images for the latent fine spatial resolution land cover map. As the convolution kernel is definite, the deconvolution process is a non-blind problem. Considering the image convolution process as shown in Equation (2), the relationship between the interpolated fine spatial resolution fraction images and the latent fine spatial resolution land cover map can be represented as:

$$
\mathbf{F}=\mathbf{X} \otimes \mathbf{H}+n
$$

where $n$ is the noise in the interpolated fine spatial resolution fraction images.

Generally, estimating $\mathbf{X}$ from $\mathbf{F}$ is not a well-conditioned problem. To obtain a stable solution, a specific regularization needs to be imposed on the observation model in Equation (5) $[\underline{36}, \underline{37]}$. The regularization takes the form of constraints in the space of possible solutions, which is often independent of measured data, and is constructed based on a priori knowledge on the spatial patterns of land cover in the fine spatial resolution land cover map. Therefore, the de-convolution process can be converted to a cost minimization function:

$$
\mathbf{X}=\arg \min _{\mathbf{x}}\left[D(\mathbf{X}, \mathbf{F})+l_{i}(\mathbf{X})\right]
$$

where $D(\mathbf{X}, \mathbf{F})$ is the data error term, $\Upsilon(\mathbf{X})$ is the regularization term, and $\lambda$ is a weight parameter that balances the contribution of regularization and data error terms.

The data error term measures the difference between the solution and the observed data, and the popular least squares cost function is applied:

$$
D(\mathbf{X}, \mathbf{F})=\|\mathbf{F}-\mathbf{H} \otimes \mathbf{X}\|_{2}^{2}
$$

The regularization term provides particular knowledge about the spatial pattern of land cover classes. In SRM, the spatial dependence principle, that is, the tendency for spatially proximate observations of a given property to be more similar to one another that distant observations, is widely used [35]. Based on this principle, the spatial regularization term is expressed as:

$$
\Upsilon(\mathbf{X})=\sum_{v=1}^{(z \times M) \times(z \times N)} \Upsilon\left(x_{v}\right)=\sum_{v=1}^{(z \times M) \times(z \times N)} \sum_{n=1}^{w \times w} \phi_{n} \times \delta\left(x_{v}, x_{n}\right)
$$

where

$$
\begin{gathered}
\delta\left(x_{v}, x_{n}\right)= \begin{cases}0 & \text { if } x_{v}=x_{n} \\
1 & \text { otherwise }\end{cases} \\
\phi_{n}=\left(d\left(x_{v}, x_{n}\right)\right)^{-\kappa}
\end{gathered}
$$

where $\delta\left(x_{v}, x_{n}\right)$ characterizes the relationship between the fine spatial resolution pixel $x_{v}$ and the neighbors $x_{n}$ within a $w \times w$ window. $\phi_{n}$ is the spatial weighting function that balances the influence of different fine spatial resolution pixel $x_{n} \cdot d\left(x_{v}, x_{n}\right)$ is the distance between $x_{v}$ and $x_{n}$, and $\kappa$ is the power law index that controls the nonlinear parameter of the distance decay model.

The simulated annealing (SA) algorithm [38] is applied to optimize the de-convolution model. The annealing schedule is based on a power law decay function, where temperature $T_{n}$ at iteration $n$ is changed to

$$
T_{n}=\sigma \cdot T_{n-1}
$$




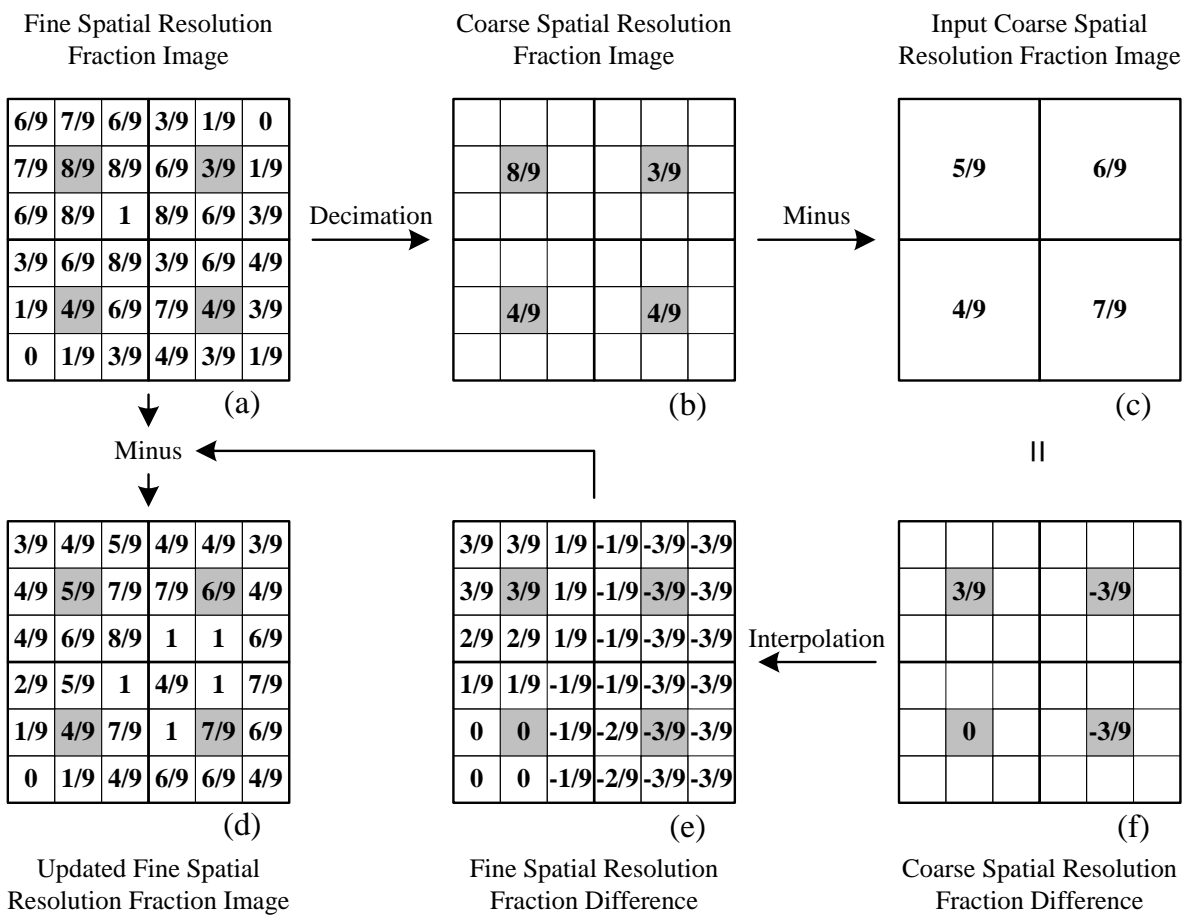

Fig. 3. The flowchart of the back projection process.

Parameter $\sigma \in(0,1)$ controls the decrease rate of temperature $T_{n}$. In the initialization step, a fine resolution land cover map is generated by assigning a random class label to each fine resolution pixel. In one iteration step, all fine spatial resolution pixels are visited using a row wise scheme. For each fine spatial resolution pixel, it is changed to another class label randomly. The changed class label is accepted if doing so can decrease the object function value in Equation (6). Otherwise, if doing so increases the object function value, the change class label is accepted only with a very low probability according to the current temperature. The iterations terminate when the previously fixed number of iterations is achieved.

\section{Re-convolution}

Initially, the fine spatial resolution land cover map produced by the de-convolution process is generally far from reality. The main reason is that most values in the convoluted fine spatial resolution fraction images estimated by interpolating observed coarse spatial resolution fraction images are different from the real values, because of the errors included in the observed fraction images and caused by the interpolation algorithm. In theory, if the fine spatial resolution land cover map $\mathbf{X}_{(k)}$ produced by de-convolution is similar to the latent fine spatial resolution land cover map, the forward model of the conceptual image formation process should be satisfied. Therefore, if the convolution and decimation process are applied to the intermediate fine spatial resolution land cover map $\mathbf{X}_{(k)}$ again, the result should be closer to the observed fraction images. Otherwise, $\mathbf{X}_{(k)}$ should be refined by using the back projection process as follows.

\section{Back Projection}

The underlying idea of back projection is that the fraction images produced from the fine spatial resolution land cover map by applying the conceptual image formation process should be identical to the observed coarse spatial resolution fraction images. In order to make them consistent, the backprojected operation is expressed as:

$$
\mathbf{F}=\mathbf{F}-\left(\mathbf{F} \downarrow^{z}-\mathbf{Y}\right) \uparrow^{z}
$$

where $\mathbf{F}$ is the original fine spatial resolution fraction image, and $\mathbf{F}$ is the updated fine spatial resolution fraction image with back projection. $\downarrow^{z}$ is the decimation operation and $\uparrow^{z}$ is the interpolation operation with the zoom factor $z$.

This procedure is illustrated in Figure 3. Fig. 3(a) shows the fine spatial resolution fraction image produced by reconvolving the intermediate fine spatial resolution land cover map. The decimation process is applied to generate a coarse spatial resolution fraction image, as shown in Fig. 3(b). Fig. 3(c) shows the observed coarse spatial resolution fraction image. Fraction values in Fig. 3 (b) and (c) are then compared and their fraction difference image is calculated, as shown in Fig. 3(f). This coarse spatial resolution fraction difference value is further interpolated to generate a fine spatial resolution fraction difference image as shown in Fig. 3(e), which is again used to update the fine spatial resolution fraction images in Fig. 3(a), and the updated result is shown in Fig. 3(d).

\section{E. The proposed algorithm}

The proposed IID algorithm is summarized as follows:

1) Interpolate the input coarse spatial resolution fraction images for each land cover class; 
2) Estimate an initial fine spatial resolution land cover map, by optimizing the object function of de-convolution expressed in Equation (6) with the simulated annealing algorithm, using the interpolated fine spatial resolution fraction images as input;

3 ) Re-convolute the fine resolution land cover map produced in the step 2);

4) Update the fine spatial resolution fraction images by backprojection, with the input coarse spatial resolution fraction images and the re-convoluted fine spatial resolution fraction images produced in the step 3).

5) Produce the fine spatial resolution land cover map with updated fine spatial resolution fraction images;

6) Repeat steps 3)-5), if the iteration time is less than the maximal time or the difference between the updated fine spatial resolution fraction images compared to that of the previous iteration is less than $0.1 \%$; otherwise, the updated fine spatial resolution land cover map is regarded as the final result.

\section{EXPERIMENTS}

\section{A. Simulated shapes}

To illustrate and evaluate the performance of the proposed algorithm, we first applied it to a set of synthetic categorical images derived from three artificial shapes including the number six, a cross, and an open triangle (Fig. 4). All original shape images were $70 \times 70$ pixels and included two classes representing the shape and the background. The coarse spatial resolution fraction images were then simulated from the original images, and three zoom factors $\mathrm{z}=5, \mathrm{z}=7$, and $\mathrm{z}=10$ were considered. At each zoom factor, the area proportions of different classes in each coarse spatial resolution pixel in a window were calculated and assigned as the fraction values. In order to simulate the effects of error introduced by spectral unmixing, Gaussian noise was added in the simulated fraction images. The root mean square error (RMSE) was used to compare the class area proportions in the simulated fraction images with those computed from original shape images, which are used as the reference. The RMSE is calculated as follows:

$$
\operatorname{RMSE}=\sqrt{\sum_{c=1}^{C} \frac{1}{M \times N} \sum_{V=1}^{M \times N}\left(y_{V, S I M}^{c}-y_{V, R E F}^{c}\right)^{2} / C}
$$

where $y_{V, S I M}^{c}$ denotes the fraction value of the $c^{\text {th }}$ class in the coarse resolution pixel $V$ in the simulated fraction images, and $y_{V, R E F}^{c}$ denotes the fraction value of the $c^{\text {th }}$ class in the coarse resolution pixel $V$ in the reference land cover map.

Three fraction images with RMSE $=0,0.05$ and 0.10 were

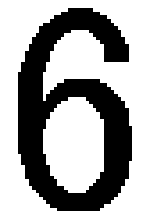

(a)

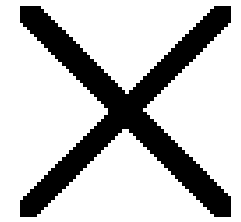

(b)

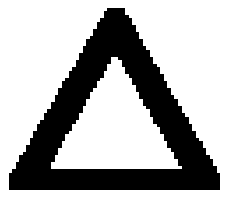

(c)
Fig. 4. The reference fine spatial resolution shapes with $70 \times 70$ pixels.

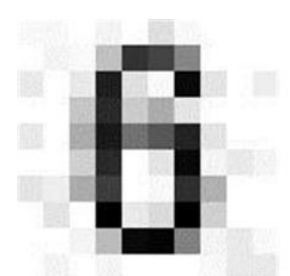

Fraction Image

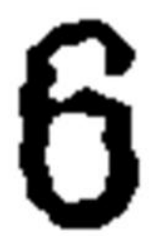

$2^{\text {nd }}$ Iteration

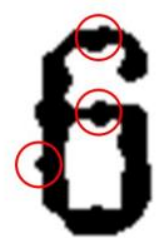

Initial

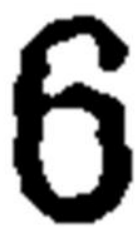

$3^{\text {rd }}$ Iteration

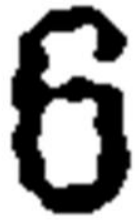

$1^{\text {st }}$ Iteration

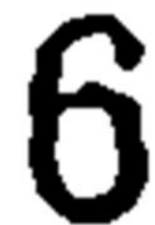

$4^{\text {th }}$ Iteration
Fig. 5. The fraction image of the shape of number six with $z=7$ and RMSE $=0.05$, and initial fine spatial resolution land cover map and updated ones at different iteration times. The initial map includes many local convex parts as indicated by circles.

simulated for each shape at each zoom factor. The simulated fraction images were then considered as the outputs of a spectral unmixing analysis and were used as the SRM input. In addition to the proposed IID algorithm, the pixel swapping algorithm (PS) [9], the bilinear interpolation based SRM algorithm (BI) [22], and the Hopfield neural network based SRM algorithm (HNN) [2] were also applied for comparison. The performance of all these algorithms was visually and quantitatively assessed on the basis of the reference fine spatial resolution land cover map. The accuracy of fine spatial resolution land cover maps produced by various algorithms was assessed using the overall accuracy value that is measured by comparing the result and reference fine spatial resolution maps.

The output of a SRM algorithm is dependent on the parameter settings used. For the PS algorithm, according to [39], the neighboring window size is set to be $5 \times 5$, and the power law index in the distance decay model is set to be one, meaning that the 24 closest fine resolution pixels with equal weights are applied to calculate the spatial dependence of a fine spatial resolution pixel. For the BI algorithm, the bilinear interpolation method was used to magnify the coarse spatial resolution fraction images, and the sequent assigning method were applied to assign class labels of fine spatial resolution pixels, in order to produce the final fine spatial resolution land cover map [22]. For the proposed IID algorithm, the bilinear interpolation algorithm was used for spatial interpolation. For the de-convolution object function in equation (6), parameters are determined by trial and error. According to our experiments, a $5 \times 5$ neighboring window in which the power law index was set to be one was suitable to calculate the regularization term in equation (8). For the weight parameter $\lambda$, it is found that the optimal value is mainly affected by fraction errors. By comparing various SRM results, the same values of $\lambda$ were used for all experiments in this paper, that is, $0.05,0.08$ and 0.10 for $\mathrm{RMSE}=0,0.05$ and 0.10 , respectively.

During an analysis with the proposed IID algorithm, an initial 


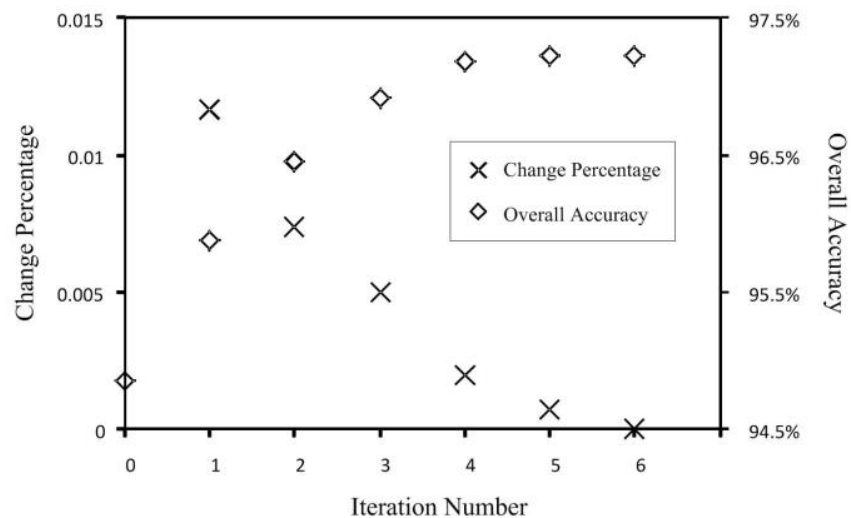

Fig. 6. The overall accuracy of each fine spatial resolution land cover map in the initial step and different iteration times, and the percent of changed fine spatial resolution pixels between two iterations for the experiment of the shape of number six with $\mathrm{z}=7$ and $\mathrm{RMSE}=0.05$.

fine spatial resolution land cover map is first produced by deconvolution, then, the initial fine spatial resolution land cover map is iteratively updated by re-convolution, back-projection and de-convolution processes. Taking the shape of number six with $\mathrm{z}=7$ and $\mathrm{RMSE}=0.05$ as example, Figure 5 shows the initial fine spatial resolution land cover map and those associated with the next four iterations. It is evident that the initial map includes many local convex parts and is different from the reference fine spatial resolution map. This is mainly caused by value errors in the initial interpolated fine spatial resolution fraction images. With the increment of iteration, the local convex parts disappear gradually, because the reinforcement of observed fraction values by back-projection can act to decrease the value errors in the fine spatial resolution fraction images. The fine spatial resolution land cover maps produced in the $3^{\text {rd }}$ and $4^{\text {th }}$ iterations are almost the same and both very similar to the reference map.

Figure 6 shows the overall accuracy of each fine spatial resolution land cover map and the percent of changed fine spatial resolution pixels between two iterations. For the $1^{\text {st }}$ iteration, the changed percent is larger than 0.01 , meaning that more than $1 \%$ pixel labels in the initial map was updated. The changed percent decreases gradually with the increment of iteration and reaches 0 in the $6^{\text {th }}$ iteration. Accordingly, the overall accuracy increases with the increment of iteration and becomes stable with the highest value in the $6^{\text {th }}$ iteration. Therefore, after six iterations, the final fine spatial resolution land cover map was produced. In this experiment, for all cases with various shapes, RMSE values, and zoom factors, the final result can be produced within 10 iterations.

Figure 7 shows the resultant fine spatial resolution land cover maps generated by the selected SRM algorithms, each using the coarse resolution fraction images simulated with RMSE $=0.05$ and $\mathrm{z}=7$ as input. In the fine spatial resolution land cover maps produced by PS, there are many speckle-like artifacts caused the fraction errors in the simulated fraction images. Likewise, the fine spatial resolution land cover maps produced by BI are also characterized by many speckle-like artifacts. Moreover, the continuous shapes are broken into several individual patches in the result of PS. In the result of BI, the spatial continuity of these shapes was better maintained, but these shapes have irregular boundaries. By contrast, the fine spatial resolution land cover maps produced by HNN and the proposed IID algorithm are similar to the reference. Most speckle-like artifacts are eliminated, and their spatial continuities are well represented by both HNN and IID. However, the shape boundaries in the result of HNN have some local convex parts, while those in the result of IID are smoother, showing that IID is superior to $\mathrm{HNN}$.

The overall accuracies of the different algorithms are shown in Table I. The results indicate that classification accuracy varied with the zoom factor and simulated fraction RMSE values. With the increment of zoom factor or the simulated
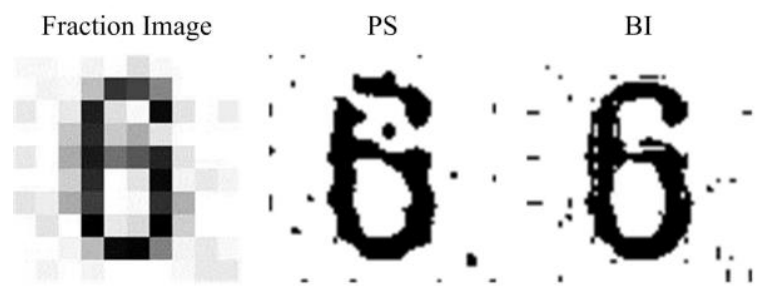

HNN

IID
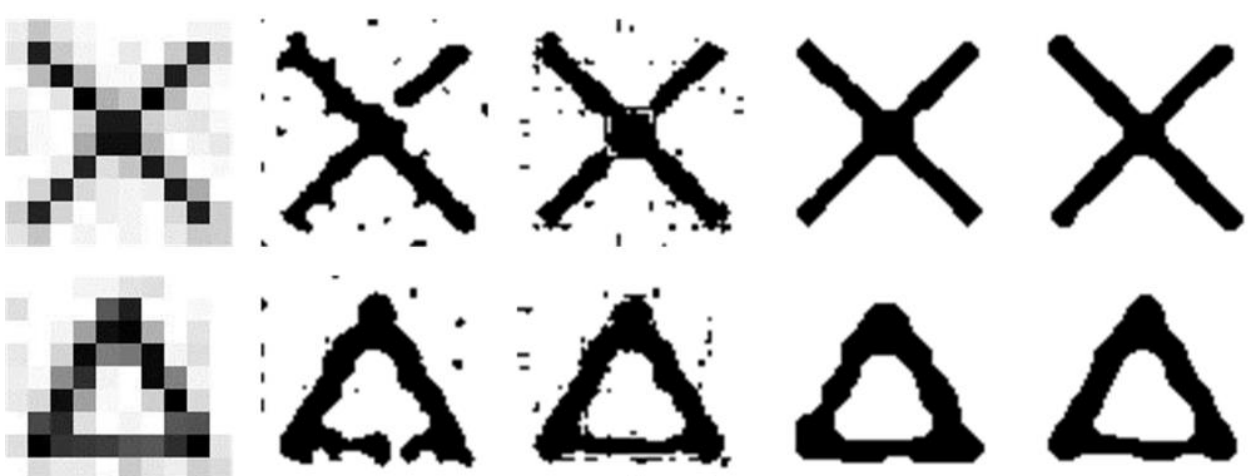

Fig. 7. Fraction images of the simulated shape experiment with $\dot{\mathrm{z}}=7$ and $\mathrm{RMSE}=0.05$, and result fine spatial resolution land cover maps generated from different SRM methods. 
TABLE I

OVERALL ACCURACIES (\%) OF THE FINE SPATIAL RESOLUTION LAND COVER MAPS PRODUCED BY THE PS, BI, HNN AND IID ALGORITHMS FOR THE SIMULATED SHAPES EXPERIMENT. THE HIGHEST OF THE 3 ACCURACY VALUES FOR EACH SETTING OF RMSE IS HIGHLIGHTED IN BOLD.

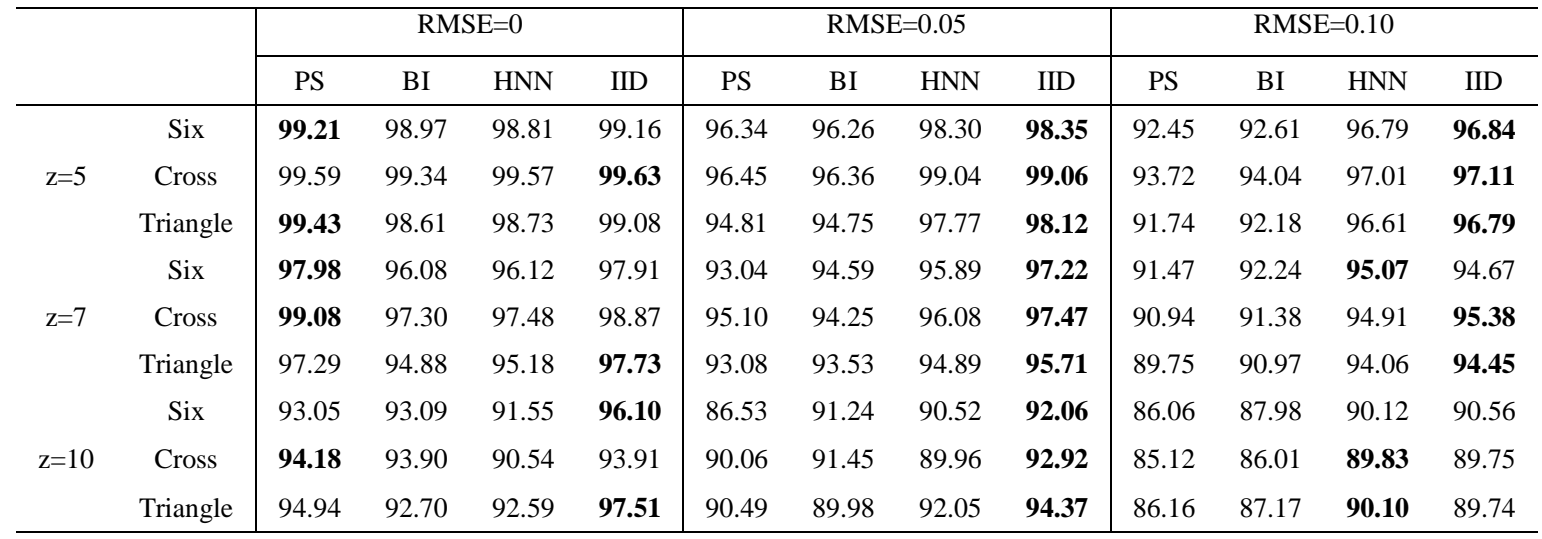

fraction RMSE value, overall accuracy tended to decrease for all cases. When the simulated fraction RMSE value was zero, which indicates that the output of the spectral unmixing was error-free, the overall accuracies of IID and PS are similar and are both higher than those of BI and HNN in most cases. For shapes of the number six and the open triangle at $\mathrm{z}=10$, however, the overall accuracies of IID are much higher than those of PS. When the simulated fraction RMSE value equals to 0.05 or 0.10 , the overall accuracies of IID and HNN are all higher than those of PS and BI. When RMSE $=0.05$, the overall accuracies of IID are all higher than those of HNN, showing the effectiveness of the proposed IID algorithm. When RMSE = 0.10 , however, there are little differences between the overall accuracies of IID and HNN. This is because a higher RMSE value introduces more errors in both interpolation and deconvolution processes, and then decreases the accuracy of the result.

\section{B. Simulated multi-spectral images}

In this experiment, a simulated multi-spectral image was used. The data were obtained from a subset of Quickbird panchromatic image in Wuhan, Hubei Province, China in Fig. 8 (at spatial resolution of $0.6 \mathrm{~m}$ ). The panchromatic image was manually interpreted to form the reference map of $120 \times 120$ pixels containing 4 classes: tree, grass, ground, and path. With this reference map, three different coarse spatial resolution multi-spectral images were simulated. The spectral band number of the simulated multi-spectral image was set as 6 , and the spatial resolution was set as $3 \mathrm{~m}$, meaning the zoom factor equals to 5 . The 4 endmember reflectance values were manually set to $[0.73,0.45,0.27,0.13,0.09,0.12]^{\mathrm{T}},[0.21,0.08,0.13$, $0.23,0.31,0.37]^{\mathrm{T}},[0.05,0.69,0.34,0.69,0.94,0.90]^{\mathrm{T}}$ and $[0.90,0.22,0.52,0.36,0.68,0.49]^{\mathrm{T}}$. The covariance matrices were defined following the approach discussed in [38]. For three different cases, the covariance matrices for all the classes were manually set to a $6 \times 6$ identity matrix multiplied by $0,0.06$ and 0.26 respectively. The spectral values of fine-resolution pixels of the simulated image were independent and identically distributed, and the fine-resolution pixels were conditionally independent given their class association. The fine-resolution

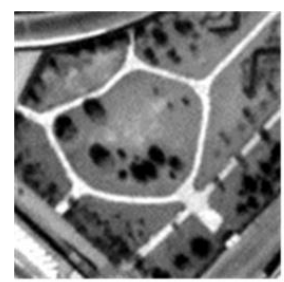

Panchromatic image

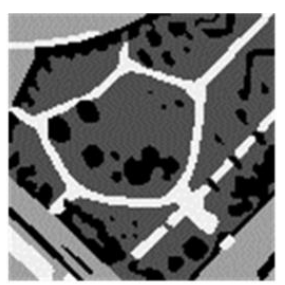

Reference map

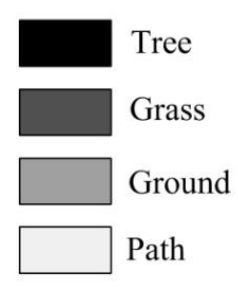

Fig. 8. The panchromatic image and reference map for the simulated multispectral image experiment.

multi-spectral image was spatially degraded to the coarseresolution multi-spectral image using a $5 \times 5$ mean filter. The linear spectral unmixing method was then applied to generate fraction images for all three cases. The resulting fraction images have the RMSE values of $0,0.05$ and 0.10 , respectively. Using the fraction images as input, PS, BI, HNN and IID algorithms were applied to produce final fine spatial resolution land cover maps.

The fine spatial resolution land cover maps shown in Fig. 9 were used to visually assess the performance of various SRM algorithms. The value of RMSE greatly affected the performance of all SRM algorithms. The quality of the final fine spatial resolution map from each SRM algorithm was negatively related to the magnitude of the RMSE; thus the smaller the RMSE, and so the better the unmixing, the more accurate the final SRM. Both the PS and BI algorithms maintain absolutely the input class fractional cover information in the resulting fine spatial resolution land cover maps. Therefore, a larger RMSE value brings more speckle-like errors in the result of PS and BI algorithms. Moreover, PS breaks continuous patches into several parts, such as the circle of the 'path' class, and the application of BI makes patch boundaries less smooth. HNN eliminates most speckle-like errors in the result; however, it also breaks many continuous land cover patches. By contrast, IID can overcome these shortcomings of PS, BI and HNN. Although large RMSE value decreases the performance of IID, no obvious speckle-like errors are found in the result produced by IID. The spatial continuity of land cover is better kept and the patch boundaries are also smoother in the result of IID. 

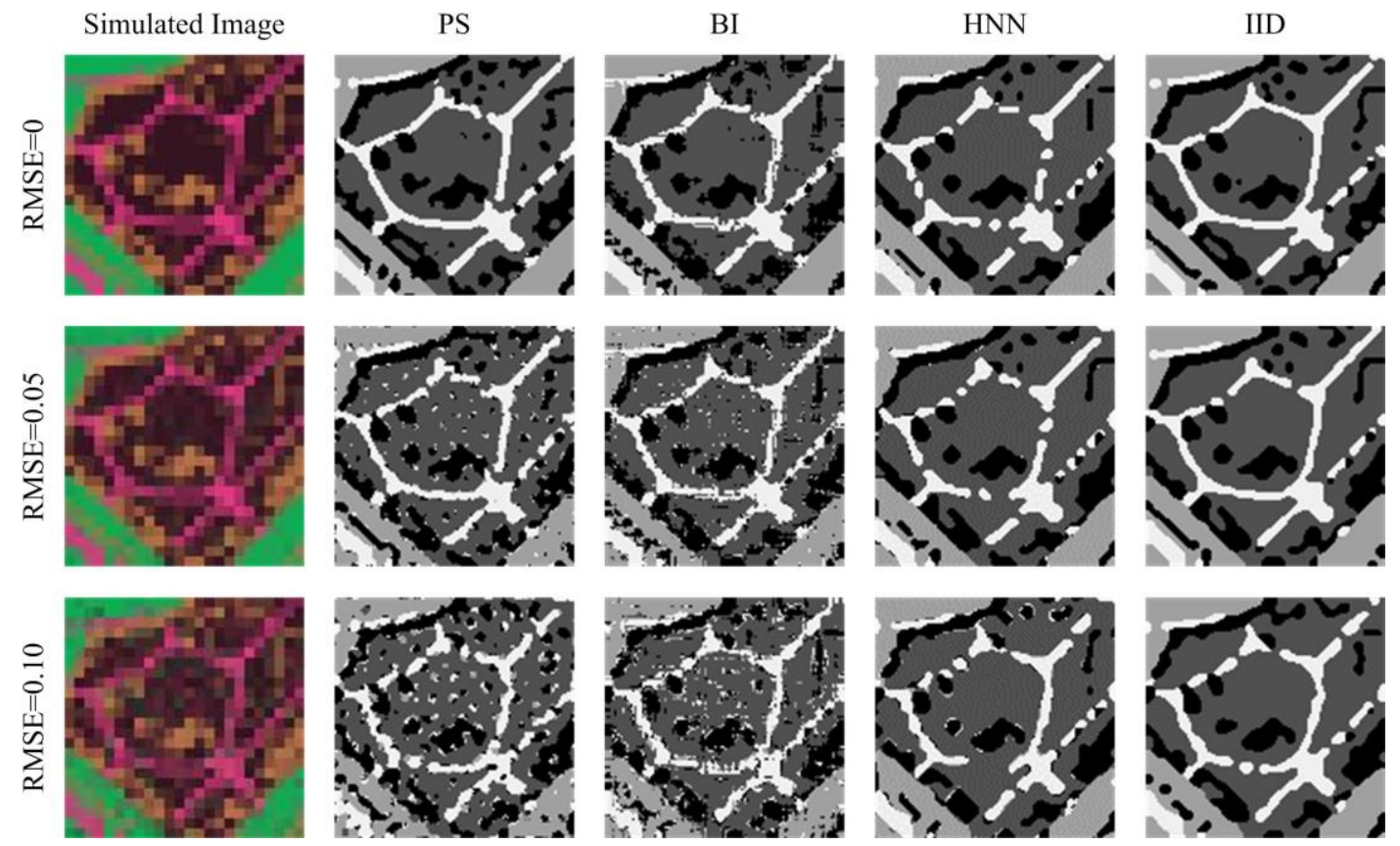

Fig. 9. Simulated multi-spectral images and result fine spatial resolution land cover maps generated from different SRM methods.

TABLE II

OVERALL ACCURACIES (\%) OF DIFFERENT SRM ALGORITHMS FOR THE SIMULATED MULTI-SPECTRAL

\begin{tabular}{ccccc}
\hline RMSE & PS & BI & HNN & IID \\
\hline 0 & 91.28 & 88.19 & 87.94 & $\mathbf{9 2 . 2 4}$ \\
0.05 & 83.53 & 83.70 & 87.83 & $\mathbf{9 0 . 5 9}$ \\
0.10 & 76.74 & 77.84 & 84.24 & $\mathbf{8 7 . 2 9}$ \\
\hline
\end{tabular}

The accuracy statistics in Table II shows an increase in the overall accuracies for IID compared with PS, BI and HNN. When RMSE $=0$, the improvement of overall accuracy for IID is $0.96 \%$ compared with PS, $4.05 \%$ compared with $\mathrm{BI}$, and $4.30 \%$ compared with HNN. The overall accuracy of PS is similar as that of BI for RMSE $=0.05$ and 0.10. Compared with PS and BI, the overall accuracy of HNN increases about $4 \%$ with RMSE $=0.05$ and $7 \%$ with $\mathrm{RMSE}=0.10$. The overall accuracy of IID is the highest, and the improvement reaches about $3 \%$, compared with that of HNN.

\section{Synthetic Landsat images}

In this experiment, the performance of the proposed IID algorithm was validated using synthetic Landsat Thematic Mapper (TM) images. A TM scene located in the Brazilian Amazon Basin and acquired on June 19, 2006 was used. The original Landsat TM image used for analysis includes bands 1, $2,3,4,5$, and 7 . The experiment was conducted with a subset of $350 \times 350$ pixels, as shown in Fig. 10(a). The original Landsat TM image was degraded to simulate coarse-resolution multispectral images at the zoom factors $\mathrm{z}=7$. The simulated coarse-resolution images are of $50 \times 50$ pixels, as shown in Fig. 10(b). The original Landsat TM image was visually interpreted to generate a fine resolution land cover map to serve as a reference data set. This map comprised four land cover classes: barren, grassland, cultivated and forest (see Fig. 10(c)). The simulated coarse-resolution image was unmixed to generate the fraction images using the linear spectral unmixing method, and the resulting RMSE is 0.097. Using the fraction images as input, fine spatial resolution land cover maps were produced by the four SRM algorithms.

The land cover maps produced by different SRM algorithms are shown in Fig. 10 and the accuracy statistics of different algorithms are shown in Table III. Both the fine spatial resolution land cover maps produced by PS and BI include many speckle-like errors, which are often small round patches for PS and linear patches for BI. BI can better maintain the continuity of land cover patches than PS, while PS produces smoother patch boundaries. HNN eliminates most speckle-like errors, but the continuity of land cover patches is not well presented. IID produces better fine resolution land cover maps than both PS, BI and HNN, as it eliminates most speckle-like errors, and preserves the patch continuity and the patch boundary smoothness at the same time. The overall accuracies of all SRM algorithms also show the effectiveness of the proposed IID algorithm. The improvement of overall accuracy is $8.17 \%, 6.63 \%$ and $1.67 \%$, compared with PS, BI and HNN, respectively.

TABLE III

OVERALL ACCURACIES (\%) OF DIFFERENT SRM ALGORITHMS FOR THE DEGRADED LANDSAT TM IMAGE

\begin{tabular}{ccccc}
\hline & PS & BI & HNN & IID \\
\hline OA & 85.86 & 87.40 & 92.36 & $\mathbf{9 4 . 0 3}$ \\
\hline
\end{tabular}




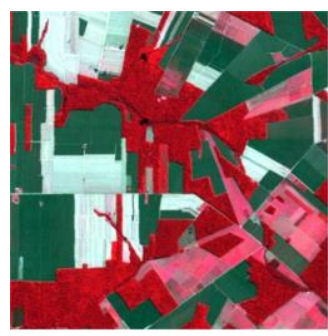

(a)

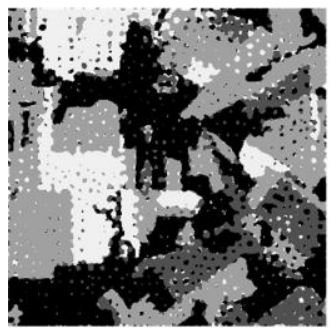

(d)

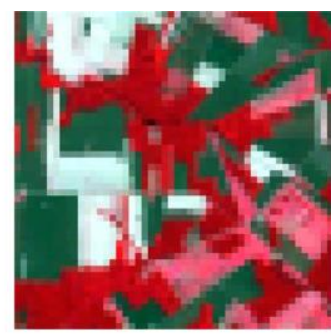

(b)

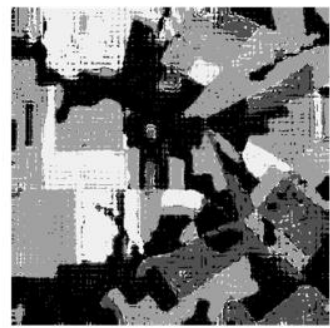

(e)

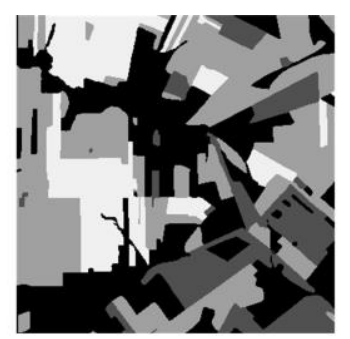

(c)

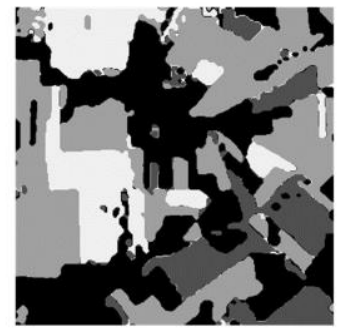

(f)
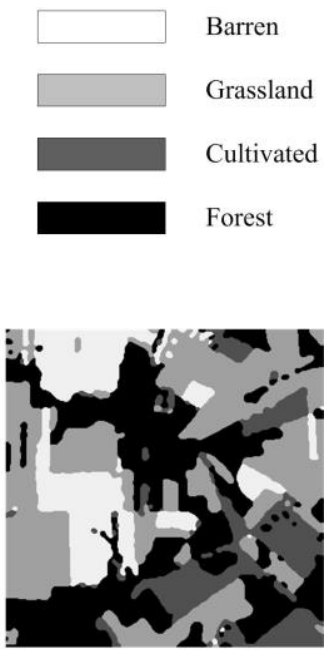

(g)

Fig. 10. Landsat TM data set and results. (a) is the Landsat TM multi-spectral image (band 4-3-2). (b) is the degraded Landsat TM images with zoom factor $\mathrm{z}=7$. (c) is the reference land cover map. (d)-(g) are land cover maps generated by pixel swapping, bilinear interpolation, Hopfield neural network and the proposed iterative interpolation de-convolution SRM algorithms, respectively.

\section{Discussion}

The experimental results confirm that the proposed IID algorithm can produce a fine spatial resolution land cover map with higher accuracy than PS, BI, and HNN algorithms. There are two main advantages of the proposed IID algorithm. First, it can eliminate the impact of fraction errors caused by spectral unmixing on the result. This feature is mainly attributed to the de-convolution process, which takes account of the fraction errors in the interpolated fine spatial resolution fraction images and suppresses its affect by spatial regularization. Second, IID can preserve the patch continuity and the patch boundary smoothness, simultaneously. The patch continuity is preserved because the interpolation process takes account of neighboring fraction values, and the resulting interpolated fine resolution fraction images are spatial smooth. The patch boundary smoothness is preserved because the spatial regularization model applies the local smoothness as the prior model of the latent fine spatial resolution land cover map.

The iterative back projection technique has been widely used in image super-resolution algorithms [40]. This kind of image super-resolution algorithm often first uses a simple bilinear or bicubic interpolation method to produce an initial fine resolution image. Then, the interpolated fine resolution image is refined by various models, such as non-local processing [41] or adaptive enhancement [42], and the back projection technique is used to minimize the reconstruction error iteratively. Compared with these image super-resolution algorithms, the proposed IID algorithm has a similar framework, as it also first interpolates the coarse spatial resolution fraction images, and then iteratively refines the result with the back projection technique. The difference is that a special de-convolution model is applied in the IID algorithm to produce the fine resolution land cover map, which has discrete
TABLE IV

RUNNING TIME OF DIFFERENT SRM ALGORITHMS FOR DIFFERENT IMAGES

\begin{tabular}{cccccc}
\hline Image & Coarse Pixels & PS & BI & HNN & IID \\
\hline Shapes & $10 \times 10$ & $10 \mathrm{sec}$ & $1 \mathrm{sec}$ & $2 \mathrm{~min}$ & $2 \mathrm{~min}$ \\
Simulated & $24 \times 24$ & $43 \mathrm{sec}$ & $2 \mathrm{sec}$ & $10 \mathrm{~min}$ & $11 \mathrm{~min}$ \\
Landsat & $50 \times 50$ & $58 \mathrm{sec}$ & $4 \mathrm{sec}$ & $114 \mathrm{~min}$ & $120 \mathrm{~min}$ \\
\hline
\end{tabular}

class labels instead of continue image values, from the interpolated and refined fine resolution fraction images.

The most important parameter in the proposed IID algorithm is the regularization value $\lambda$. The regularization parameter acts as a tradeoff parameter that balances the influence of the data fidelity and regularization terms on the solution of (6). If the regularization parameter is too small, the solution is unsmoothed and susceptible to the noise in input interpolated fine spatial resolution fraction images. If it is too large, the regularization term has a dominant effect on the solution, generating an over-smoothed fine spatial resolution land cover map. The data fidelity and regularization terms should be appropriately balanced when the de-convolution process is implemented. Here, through trial and error, the regularization value is set to be 0.05 for $\mathrm{RMSE}=0,0.08$ for $\mathrm{RMSE}=0.05$, and 0.10 for $\mathrm{RMSE}=0.10$. As the de-convolution model is based on the regularization theory, some popular methods, such as Lcurve and U-curve $[\underline{35}, \underline{40}]$, can also be used to select the optimal value of the regularization parameter.

All algorithms were tested on an Intel Core i7-4770 3.40 GHz CPU with 16.0-GB RAM using MATLAB version R2012a. For HNN, the iteration number was set to be 3000 . For IID, the iteration number was set to be 8 , and the inner iteration number in the de-convolution step was set to be 70 . The run times of 
different SRM algorithms are listed in Table IV. Run time increases with image size for different SRM algorithms. BI is the fastest algorithm, and PS also converges within a short run time. The run times of HNN and IID are about the same, and are both longer than those of BI and PS, because they need the iteration process to eliminate the impact of fraction errors caused by spectral unmixing on the result.

Although the proposed IID algorithm has shown its effectiveness, some further improvements could be made. First, as the quality of the initial interpolated fine spatial resolution fraction images impact the subsequent iterations and the final result, there may be value in exploring the potential of different interpolation methods. In this paper, the bilinear interpolation method was applied and it could be replaced by some advanced interpolation algorithm. Second, in the de-convolution object function, the spatial smoothness principle was applied for regularization. However, the performance would be expected to vary with the nature of the land cover mosaic. Therefore, some special regularization terms, such as an anisotropic land cover prior, should sometimes be adopted as the regularization term to better preserve detailed information on land cover patches. Finally, when the regularization parameter is set, a high-weight regularization parameter should be used in homogeneous regions to better remove speckle-like artifacts, while a lowweight regularization parameter should be used near patch boundaries to better preserve boundary shapes. In this present research, a fixed regularization parameter was applied and it should perhaps be replaced with a locally adaptive method for regularization parameter estimation.

\section{CONCLUSION}

A novel iterative interpolation de-convolution algorithm was proposed for super-resolution land cover mapping. Through analyzing the popular interpolation-based two-step SRM algorithms, we found that the MAP principle, which is used as the second step in existing algorithms, is suitable only when an area-to-point interpolation method is used. If a traditional areato-area interpolation method is used, the MAP process should be replaced by the de-convolution process, based on a new constructed conceptual image formation process that establishes the relationship between the observed coarse spatial resolution fraction images and the latent fine spatial resolution land cover map. The proposed IID algorithm first interpolates the input coarse spatial resolution fraction images to fine spatial resolution, and then produces an initial fine spatial resolution land cover map by a de-convolution process. This fine spatial resolution land cover map is further updated iteratively by reconvolution, back-projection and de-convolution until the final fine spatial resolution land cover map is produced.

The performance of the proposed IID algorithm was assessed with several experiments including simulated shapes, simulated multi-spectral images, and spatially degraded Landsat images. The proposed IID algorithm was compared with the popular PS, $\mathrm{BI}$ and HNN based SRM algorithms. The results show that the proposed IID algorithm produced fine spatial resolution land cover maps with higher accuracies than those produced by PS, BI and HNN algorithms. The proposed IID algorithm can reduce the impact of fraction errors caused by spectral unmixing on the resulting fine spatial resolution land cover map. Moreover, the proposed IID algorithm can preserve the patch continuity and patch boundary smoothness simultaneously, because the interpolation process uses neighboring coarse spatial resolution fraction values and the deconvolution process used the local smoothness of land cover patches as the prior information. In practice, the performance of the proposed IID depends on the interpolation algorithm and the object function of the de-convolution process. Some potential further improvements about the IID algorithm, including the spatial interpolation algorithm, the spatial regularization term and the regularization parameter estimation method in the deconvolution object function, were highlighted.

\section{REFERENCES}

[1] P. M. Atkinson, "Downscaling in remote sensing," International Journal of Applied Earth Observation and Geoinformation, vol. 22, pp. 106-114, Jun 2013.

[2] A. J. Tatem, H. G. Lewis, P. M. Atkinson, and M. S. Nixon, "Superresolution target identification from remotely sensed images using a Hopfield neural network," IEEE Transactions on Geoscience and Remote Sensing, vol. 39, pp. 781-796, 2001.

[3] G. M. Foody, A. M. Muslim, and P. M. Atkinson, "Super-resolution mapping of the waterline from remotely sensed data," International Journal of Remote Sensing, vol. 26, pp. 5381-5392, Dec 2005.

[4] J. P. Ardila, V. A. Tolpekin, W. Bijker, and A. Stein, "Markov-randomfield-based super-resolution mapping for identification of urban trees in VHR images," ISPRS Journal of Photogrammetry and Remote Sensing, vol. 66, pp. 762-775, 2011.

[5] F. Ling, X. D. Li, F. Xiao, S. M. Fang, and Y. Du, "Object-based subpixel mapping of buildings incorporating the prior shape information from remotely sensed imagery," International Journal of Applied Earth Observation and Geoinformation, vol. 18, pp. 283-292, 2012.

[6] A. M. Muad and G. M. Foody, "Super-resolution mapping of lakes from imagery with a coarse spatial and fine temporal resolution," International Journal of Applied Earth Observation and Geoinformation, vol. 15, pp. 79-91, Apr 2012.

[7] C. Huang, Y. Chen, and J. P. Wu, "DEM-based modification of pixelswapping algorithm for enhancing floodplain inundation mapping," International Journal of Remote Sensing, vol. 35, pp. 365-381, Jan 2014.

[8] K. C. Mertens, L. P. C. Verbeke, E. I. Ducheyne, and R. R. De Wulf, "Using genetic algorithms in sub-pixel mapping," International Journal of Remote Sensing, vol. 24, pp. 4241-4247, Nov 2003.

[9] P. M. Atkinson, "Sub-pixel target mapping from soft-classified remotely sensed imagery," Photogrammetric Engineering and Remote Sensing, vol. 71, pp. 839-846, Jul 2005.

[10] T. Kasetkasem, M. K. Arora, and P. K. Varshney, "Super-resolution land cover mapping using a Markov random field based approach," Remote Sensing of Environment, vol. 96, pp. 302-314, Jun 2005.

[11] M. Q. Nguyen, P. M. Atkinson, and H. G. Lewis, "Superresolution mapping using a Hopfield neural network with fused images," IEEE Transactions on Geoscience and Remote Sensing, vol. 44, pp. 736-749, Mar 2006.

[12] A. Robin, L. Hegarat-Mascle, and L. Moisan, "Unsupervised subpixelic classification using coarse-resolution time series and structural information," IEEE Transactions on Geoscience and Remote Sensing, vol. 46, pp. 1359-1374, 2008.

[13] Y. Ge, S. Li, and V. C. Lakhan, "Development and testing of a subpixel mapping algorithm," IEEE Transactions on Geoscience and Remote Sensing, vol. 47, pp. 2155-2164, Jul 2009.

[14] F. Ling, Y. Du, F. Xiao, H. P. Xue, and S. J. Wu, "Super-resolution landcover mapping using multiple sub-pixel shifted remotely sensed images," International Journal of Remote Sensing, vol. 31, pp. 50235040, 2010.

[15] F. Ling, W. B. Li, Y. Du, and X. D. Li, "Land cover change mapping at the subpixel scale with different spatial-resolution remotely sensed imagery," IEEE Geoscience and Remote Sensing Letters, vol. 8, pp. 182186, 2011. 
[16] A. M. Muad and G. M. Foody, "Impact of land cover patch size on the accuracy of patch area representation in HNN-based super resolution mapping," IEEE Journal of Selected Topics in Applied Earth Observations and Remote Sensing, vol. 5, pp. 1418-1427, Oct 2012.

[17] Y. F. Su, G. M. Foody, A. M. Muad, and K. S. Cheng, "Combining pixel swapping and contouring methods to enhance super-resolution mapping," IEEE Journal of Selected Topics in Applied Earth Observations and Remote Sensing, vol. 5, pp. 1428-1437, Oct 2012.

[18] Y. Ge, "Sub-pixel land-cover mapping with improved fraction images upon multiple-point simulation," International Journal of Applied Earth Observation and Geoinformation, vol. 22, pp. 115-126, Jun 2013.

[19] Y. Ge, Y. H. Chen, S. P. Li, and Y. Jiang, "Vectorial boundary-based subpixel mapping method for remote-sensing imagery," International Journal of Remote Sensing, vol. 35, pp. 1756-1768, Mar 2014.

[20] X. D. Li, F. Ling, Y. Du, and Y. H. Zhang, "Spatially adaptive superresolution land cover mapping with multispectral and panchromatic images," IEEE Transactions on Geoscience and Remote Sensing, vol. 52, pp. 2810-2823, May 2014.

[21] X. Xu, Y. F. Zhong, and L. P. Zhang, "Adaptive subpixel mapping based on a multiagent system for remote-sensing imagery," IEEE Transactions on Geoscience and Remote Sensing, vol. 52, pp. 787-804, Feb 2014.

[22] F. Ling, Y. Du, X. D. Li, W. B. Li, F. Xiao, and Y. H. Zhang, "Interpolation-based super-resolution land cover mapping," Remote Sensing Letters, vol. 4, pp. 629-638, Jul 2013.

[23] Q. M. Wang, W. Z. Shi, and L. G. Wang, "Allocating classes for softthen-hard subpixel mapping algorithms in units of class," IEEE Transactions on Geoscience and Remote Sensing, vol. 52, pp. 29402959, May 2014.

[24] J. Verhoeye and R. De Wulf, "Land cover mapping at sub-pixel scales using linear optimization techniques," Remote Sensing of Environment, vol. 79, pp. 96-104, Jan 2002.

[25] K. C. Mertens, B. De Baets, L. P. C. Verbeke, and R. R. De Wulf, "A subpixel mapping algorithm based on sub-pixel/pixel spatial attraction models," International Journal of Remote Sensing, vol. 27, pp. 32933310, Aug 2006.

[26] A. Boucher, P. C. Kyriakidis, and C. Cronkite-Ratcliff, "Geostatistical solutions for super-resolution land cover mapping," IEEE Transactions on Geoscience and Remote Sensing, vol. 46, pp. 272-283, Jan 2008.

[27] H. Jin, G. Mountrakis, and P. Li, "A super-resolution mapping method using local indicator variograms," International Journal of Remote Sensing, vol. 33, pp. 7747-7773, 2012.

[28] Q. M. Wang and W. Z. Shi, "Utilizing multiple subpixel shifted images in subpixel mapping with image interpolation," IEEE Geoscience and Remote Sensing Letters, vol. 11, pp. 798-802, Apr 2014.

[29] Y. H. Zhang, Y. Du, F. Ling, S. M. Fang, and X. D. Li, "Example-based super-resolution land cover mapping using support vector regression," IEEE Journal of Selected Topics in Applied Earth Observations and Remote Sensing, vol. 7, pp. 1271-1283, Apr 2014.

[30] G. M. Foody, "Relating the land-cover composition of mixed pixels to artificial neural network classification output," Photogrammetric Engineering and Remote Sensing, vol. 62, pp. 491-498, 1996.

[31] J. Li and A. D. Heap, "Spatial interpolation methods applied in the environmental sciences: A review," Environmental Modelling \& Software, vol. 53, pp. 173-189, 2014.

[32] L. Wang, Z. Wang, Z. Dou, and Y. Wang, "Edge-directed interpolationbased sub-pixel mapping," Remote Sensing Letters, vol. 4, pp. 11951203, 2013.

[33] Y. Chen, Y. Ge, and D. Song, "Superresolution Land-Cover Mapping Based on High-Accuracy Surface Modeling," IEEE Geoscience and Remote Sensing Letters, vol. 12, pp. 2516-2520, 2015.

[34] F. Ling, S. M. Fang, W. B. Li, X. D. Li, F. Xiao, Y. H. Zhang, et al., "Postprocessing of interpolation-based super-resolution mapping with morphological filtering and fraction refilling," International Journal of Remote Sensing, vol. 35, pp. 5251-5262, 2014.

[35] F. Ling, X. D. Li, F. Xiao, and Y. Du, "Superresolution land cover mapping using spatial regularization," IEEE Transactions on Geoscience and Remote Sensing, vol. 52, pp. 4424-4439, Jul 2014.

[36] W. Dong, L. Zhang, G. Shi, and X. Wu, "Image deblurring and superresolution by adaptive sparse domain selection and adaptive regularization," IEEE Transactions on Image Processing, vol. 20, pp. 1838-1857, 2011.

[37] W. Cao, J. Sun, and Z. Xu, "Fast image deconvolution using closed-form thresholding formulas of regularization," Journal of Visual Communication and Image Representation, vol. 24, pp. 31-41, 2013.

[38] V. A. Tolpekin and A. Stein, "Quantification of the effects of land-cover- class spectral separability on the accuracy of Markov-random-fieldbased superresolution mapping," IEEE Transactions on Geoscience and Remote Sensing, vol. 47, pp. 3283-3297, 2009.

[39] Y. Makido and A. Shortridge, "Weighting function alternatives for a subpixel allocation model," Photogrammetric Engineering and Remote Sensing, vol. 73, pp. 1233-1240, Nov 2007.

[40] V. Bannore and L. Swierkowski, "Fast Iterative Super-Resolution for Image Sequences," in 9th Biennial Conference of the Australian Pattern Recognition Society on Digital Image Computing Techniques and Applications, 2007, pp. 286-293.

[41] W. Dong, L. Zhang, G. Shi, and X. Wu, "Nonlocal back-projection for adaptive image enlargement," in 2009 16th IEEE International Conference on Image Processing (ICIP), 2009, pp. 349-352.

[42] C.-K. Lin, Y.-H. Wu, J.-F. Yang, and B.-D. Liu, "An iterative enhanced super-resolution system with edge-dominated interpolation and adaptive enhancements," EURASIP Journal on Advances in Signal Processing, vol. 9, 2015.

[43] Y. F. Zhong, Y. Y. Wu, L. P. Zhang, and X. Xu, "Adaptive MAP sub-pixel mapping model based on regularization curve for multiple shifted hyperspectral imagery," ISPRS Journal of Photogrammetry and Remote Sensing, vol. 96, pp. 134-148, Oct 2014. 